\title{
Maxillary Sinus Adenocarcinoma
}

National Cancer Institute

\section{Source}

National Cancer Institute. Maxillary Sinus Adenocarcinoma. NCI Thesaurus. Code C6240.

An adenocarcinoma that arises from the maxillary sinus. 